\title{
Second-order efficiency of fully sequential designs for estimating the product of two means with application in reliability estimation
}

\begin{abstract}
For estimating the product of two means from the general one parameter exponential family, we consider a fully Bayesian approach with conjugate priors. We derive a sharp lower bound for the Bayes Risk. We also propose a fully sequential design with an incurred Bayes Risk near the second order lower bound. An application to reliability estimation is performed analytically and through Monte Carlo simulation.
\end{abstract}

Keywords: Bayesian estimation, sequential designs, second-order optimality, oneparameter exponential family, reliability estimation
Volume 8 Issue 3 - 2019

\author{
Xing Xia, Kamel Rekab \\ Department of Mathematics and Statistics, University of \\ Missouri-Kansas City, USA
}

\begin{abstract}
Correspondence: Kamel Rekab, Department of Mathematics and Statistics, University of Missouri-Kansas City, Kansas City, MO, USA, Tel 8162694432,Email rekabk@umkc.edu
\end{abstract}

Received: April 30, 2019 | Published: May 13, 2019

\section{Introduction}

Solving estimation problems using a fully Bayesian approach for the one-parameter exponential family with conjugate priors for sequential designs was first investigated by Shapiro and Wardrop and developed for linear combinations of means thoroughly by Rekab et al. (2013) and many others. ${ }^{1,2}$

For estimating the product of means of two independent Bernoulli populations with Beta priors which has an application in estimating the reliability of a series system, Rekab \& Song $^{3}$ have derived the first order lower bound for the Bayes Risk and proposed a sequential design that achieves it.

The first-order efficiency for any sequential procedure, $\rho,{ }^{3}$ is

$$
R(p) \geq \frac{E\left[\sum_{\mathrm{i}=1}^{\mathrm{k}} \sqrt{\psi\left(\eta_{l}\right)}\left|\prod_{j \neq i}^{k} \psi\left(\eta_{j}\right)\right|\right]^{2}}{T}+o\left(\frac{1}{T}\right) .
$$

In this article, we will derive a sharper lower bound for the Bayes Risk and propose a sequential design that will achieve it at least asymptotically. Such a design will be referred to as second order efficient design.

The problem of estimating system reliability is the same as estimating the product of means of independent Bernoulli populations. We use independent Beta priors for the means and propose a sequential design that is second order efficient, it converges faster to the optimal ratio than the first order designs. Second order sequential designs are sought and show the optimality of the fully sequential design through an application of reliability estimation using Monte Carlo simulation.

\section{Second-order efficiency}

The density function of one-parameter exponential family

$$
f_{\eta_{i}}(x)=\exp \left\{\eta_{i} x-\varphi\left(\eta_{i}\right)\right\} \mathrm{d} \wedge(\mathrm{x}),-\infty<x<\infty, \eta_{i} \in \Omega,(2.1)
$$

where $\eta_{i}=\eta\left(\theta_{i}\right)$ is real-valued function, $\varphi$ is a continuously differentiable, real-valued function, $\wedge$ is a non-degenerate sigmafinite measure and $\Omega=(\eta, \bar{\eta}),-\infty<\eta<\bar{\eta}<\infty$, is a non-empty open interval, $i=1,2$. Using the square ${ }^{-}$error loss, and by adopting the Bayesian approach, the conjugate prior $\pi\left(\theta_{i}\right)$ and the posterior density $\pi\left(\theta_{i} \mid X_{1}, X_{2}\right)$ are both the one-parameter exponential family. The prior density as derived by Diaconis \& Ylvisaker. ${ }^{4}$

$$
\pi\left(\theta_{i}\right)=\frac{\exp \left\{r_{i, 0} \mu_{i, 0} \eta_{i}-r_{i, 0} \psi\left(\eta_{i}\right)\right\}}{C\left(r_{i, 0}, \mu_{i, 0}\right)}, \mu_{i} \in \Omega
$$

Where

$$
C\left(r_{i, 0}, \mu_{i, 0}\right)=\int \exp \left\{r_{i, 0} \mu_{i, 0} \eta_{i}-r_{i, 0} \psi\left(\eta_{i}\right)\right\} d \theta_{i}
$$

Consider the problem of allocating a fixed total number of observations from the independent populations, where $T=M_{1}+M_{2}$, $M_{i}$ is the sample size of component $i, i=1,2$.

The Bayes Risk for estimating the product of two means

$$
R(p)=E\left[\frac{E\left[\psi^{\prime \prime}\left(\eta_{1}\right) \mid \mathcal{F}_{T}\right] E^{2}\left[\psi^{\prime}\left(\eta_{2}\right) \mid \mathcal{F}_{T}\right]}{M_{1}}+\frac{E\left[\psi^{\prime \prime}\left(\eta_{2}\right) \mid \mathcal{F}_{T}\right] E^{2}\left[\psi^{\prime}\left(\eta_{1}\right) \mid \mathcal{F}_{T}\right]}{M_{2}}+\frac{\left[\psi^{\prime \prime}\left(\eta_{1}\right) \mid \mathcal{F}_{T}\right] E\left[\psi^{\prime \prime}\left(\eta_{2}\right) \mid \mathcal{F}_{T}\right]}{M_{1} M_{2}}\right] .
$$

where $\mathcal{F}_{T}$ is the sigma algebra generated by a total of $\mathrm{T}$ observations.

To ease the notation, let $D_{i}=E\left[\psi^{\prime \prime}\left(\eta_{i}\right)\right] ; C_{i}=E^{2}\left[\psi^{\prime}\left(\eta_{i}\right)\right], i=1,2$.

Next, we derive the second order lower bound for the Bayes Risk.
Theorem 2.1: For any sequential procedure, $p_{s}$ that satisfies the following conditions:

$$
\begin{aligned}
& M_{i} \rightarrow \infty \text { in probability, as } T \rightarrow \infty, \\
& \frac{M_{i}}{T} \rightarrow \frac{\sqrt{\psi^{\prime \prime}\left(\eta_{i}\right)} \psi^{\prime}\left(\eta_{i}\right)}{\sum_{i=1}^{2} \sqrt{\psi^{\prime \prime}\left(\eta_{i}\right)} \psi^{\prime}\left(\eta_{i}\right)} \text { in probability, as } T \rightarrow \infty,
\end{aligned}
$$




$$
R\left(p_{s}\right) \geq \frac{E\left[\left(\sqrt{D_{1} C_{2}}+\sqrt{D_{2} C_{1}}\right)^{2}\right]}{T}\left(1+\frac{E\left[\sqrt{\frac{D_{1} D_{2}}{C_{1} C_{2}}}\right]}{T}\right)+O\left(\frac{1}{T^{2}}\right)
$$

Proof: The first two terms establish the first order lower bound for the Bayes Risk, whereas the third term establishes the second order lower bound for the Bayes Risk.

$$
E\left[\frac{E\left[\psi^{\prime \prime}\left(\eta_{1}\right) \mid \mathcal{F}\right] E\left[\psi^{\prime \prime}\left(\eta_{2}\right) \mid \mathcal{F}\right]}{M_{1} M_{2}}\right]=\frac{1}{T^{2}} E\left[\frac{T}{M_{1}} \frac{T}{M_{2}}\left[\psi^{\prime \prime}\left(\eta_{1}\right) \mid \mathcal{F}\right] E\left[\psi^{\prime \prime}\left(\eta_{2}\right) \mid \mathcal{F}\right]\right] \text {. }
$$

\section{Reliability estimation of series system with two independent components}

Consider a series system with two independent components with unknown system reliability. The problem is to determine the optimal number units to be tested from each component. Each tested unit can be consideredaBernoullitrial. That is, suppose $X_{i}=\left\{X_{i 1}, \ldots, X_{i M_{i}}\right\} \sim \operatorname{Ber}\left(\theta_{i}\right)$ and $\theta_{i} \sim \operatorname{Beta}\left(a_{i}, b_{i}\right)$, where $0 \leq \theta_{\mathrm{i}} \leq 1, i=1,2$.

Then the Bayes Risk incurred after $\mathrm{T}$ units have been tested is:

$$
R\left(p_{s}\right)=\frac{E\left[\left(\sqrt{\theta_{1}\left(1-\theta_{1}\right)} \theta_{2}+\sqrt{\theta_{2}\left(1-\theta_{2}\right)} \theta_{1}\right)^{2}\right]}{T}\left(1+\frac{E\left[\frac{\sqrt{\theta_{1}\left(1-\theta_{1}\right)} \sqrt{\theta_{1}\left(1-\theta_{1}\right)}}{\theta_{1} \theta_{2}}\right]}{T}\right)
$$

as the second-order lower bound of Bayes Risk.

We also need to rely on the optimal ratio indicated in Theorem 2.1 , that is

$$
C=\frac{\sqrt{\theta_{1}\left(1-\theta_{1}\right)} \theta_{2}}{\sqrt{\theta_{1}\left(1-\theta_{1}\right)} \theta_{2}+\sqrt{\theta_{2}\left(1-\theta_{2}\right)} \theta_{1}}
$$

We proceed with the test as follows:

Step 1: Collect one sample case $\left(m_{1}=n_{1}=1\right)$ from each component, $\mathcal{P}_{1}$ and $\mathcal{P}_{2}$.

Step 2: We collect $m_{\ell}$ sample cases from component, $\mathcal{P}_{1}$ where $\ell \geq 2$,

If

$$
\frac{m_{\ell+1}+r_{1, o}}{T}<\hat{C}\left(m_{\ell+1}, n_{\ell+1}\right)
$$

where $\hat{C}=E[C \mid \mathcal{F}]$. Then $m_{\ell+1}=m_{\ell}+1$, where $m_{\ell+1}$ are the cumulative sample cases from component $\mathcal{P}_{1}$;

Otherwise, $n_{\ell+1}=n_{\ell}+1$, where $n_{\ell+1}$ is the cumulative sample cases from component $\mathcal{P}_{2}$.

Step 3: We stop the iteration in step 2 when $T=m_{\ell+1}+n_{\ell+1}$, which is the fixed total sample size.

It should be noted that $C=\frac{1}{2}$ when both components have equal prior. The following example through Monte Carlo simulation with 5000 iterations shows that the expected number of units to be tested from each component is approximately $\frac{T}{2}$ which agrees with (3.2) (Table 1)

Next, we establish the rate of convergence of $\Delta=R(p)-R\left(p_{s}\right)$. Let the speed be defined as $T^{\beta} \cdot \Delta, 0 \leq \beta<2$. It is clear that $T \cdot \Delta \rightarrow 0$ . See Table 2. It is also clear that $T^{\beta} \cdot \Delta$ is bounded as $T \rightarrow \infty$, where $\theta_{i} \sim \operatorname{Beta}\left(a_{i}, b_{i}\right)$ (Figure 1). ${ }^{6-17}$

Table I Fully sequential sampling design with uniform priors

$\begin{array}{llllllllll}\boldsymbol{T} & 50 & 70 & 100 & 200 & 400 & 600 & 800 & 1000 & 3000 \\ \mathbf{E}\left[\mathbf{m}_{1}\right] & 26 & 36 & 51 & 101 & 200 & 301 & 400 & 498 & 1504 \\ \mathbf{E}\left[\mathbf{m}_{2}\right] & 24 & 34 & 49 & 99 & 200 & 299 & 400 & 502 & 1496\end{array}$

Table $2 T^{\beta} . \Delta<C$ as $t \rightarrow \infty$, where $\Delta=R(p)-R\left(p_{s}\right), 0 \leq \beta<2, C \in \mathbb{R}$

\begin{tabular}{llllllllll}
\hline $\boldsymbol{T}$ & 50 & 70 & 100 & 200 & 400 & 600 & 800 & 1000 & 3000 \\
$\boldsymbol{T} \Delta$ & 0.02 & 0.019 & 0.026 & 0.029 & 0.033 & 0.025 & 0.034 & 0.031 & 0.032 \\
$\boldsymbol{T}^{1.5} . \Delta$ & 0.138 & 0.159 & 0.262 & 0.415 & 0.662 & 0.619 & 0.955 & 0.969 & 1.776 \\
$\boldsymbol{T}^{2} . \Delta$ & 0.976 & 1.329 & 2.62 & 5.863 & 13.231 & 15.169 & 27.001 & 30.627 & 97.281 \\
\hline
\end{tabular}

\section{Conclusion}

The proposed sequential design enables the experimenter to determine the optimal allocation of units to be tested from each component when component is functional independently. Rekab ${ }^{5}$ used a classical approach to estimate the reliability of a series system, and proposed a sequential design that was shown to be first-order optimal, that is $T \cdot \Delta \rightarrow 0$. The fully Bayesian approach enables us to investigate a design with a converge rate of the order of $T^{-\beta}$, where $0 \leq \beta<2$.

\section{Data availability}

The data used to support the findings of this study have been produced by Monte Carlo simulation from Bernoulli trials with 5000 replications. 


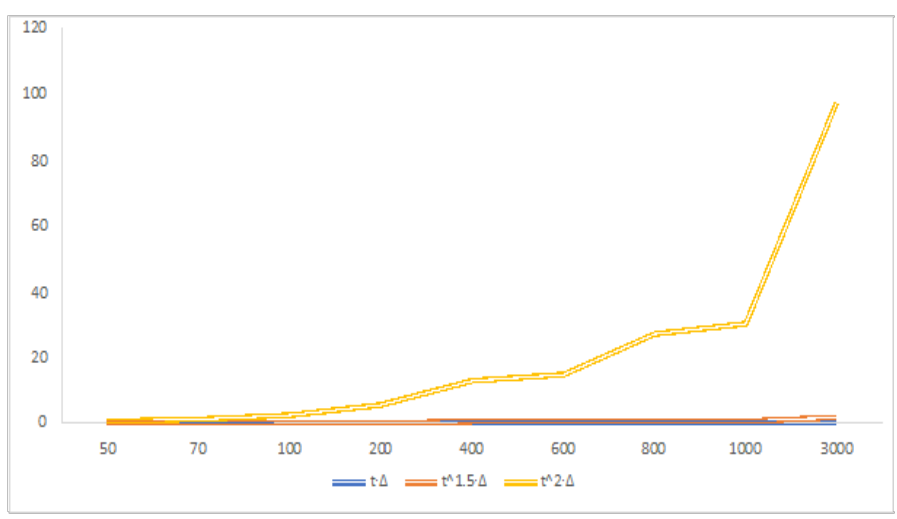

Figure I $\Delta=R(p)-R\left(p_{s}\right)$ is bounded by $T^{-\beta}$ as $T \rightarrow \infty$, where $0 \leq \beta<2$.

\section{Acknowledgements}

None.

\section{Conflicts of interest}

Authors declare that there are no conflicts of interest.

\section{References}

1. Benkamra ZT. Bayesian sequential estimation of the reliability of a parallel-series system. Applied Mathematics and Computation. 2013;209(23):10842-10852.

2. Song XRK. Second Order Optimality of Sequential Designs with Application in Software Reliability Estimation. Biom Biostat Int J. 2015;2(4):00037.

3. Rekab K, Song X. Asymptotic Efficiency in Sequential Designs for Estimating Product of $\mathrm{k}$ Means in the Exponential Family Case. Journal of Applied Mathematics and Statistics. 2017;4(1):50-69.

4. Diaconis P, Ylvisaker D. Conjugate priors for exponential families. The Annals of Statistics. 1979;7(2):269-281.
5. Rekab K. A Sampling Scheme for Estimating the Reliability of a Series System. IEEE Transactions on Reliability. 1993;287-290.

6. Ash RB. Real Analysis and Probability. Birnbaum, editors. USA: Academic Press; 1972.

7. Billingsley P. Probability and Measure. USA: John Wiley \& Sons; 2012.

8. Billinton R, Allan RN. Reliability Evaluation of Engineering Systems, Concepts and Techniques. New York: Springer; 1992.

9. Hard Wick JA. Optimal Few-Stage Designs. Journal of Statistical Planning and Inference. 2002;104:121-145.

10. Littlewood B, Wright D. Some conservative stopping rules for the operational testing of safety critical software. IEEE Transactions on Software Engineering. 1997;23(11):673-683.

11. Poore JH, Mills HD, Mutchler D. Planning and certifying software system reliability. IEEE Software. 1193;10(1):88-99.

12. Rekab K. Asymptotic Optimality of Experimental Designs in Estimating a Product of Means. Journal of Applied Mathematics and Stochastic Analysis. 1990;3(1):15-25.

13. Rekab K. A nearly optimal 2-stage procedure. Communications in Statistics-Theory and Methods. 1992;21(1):197-201.

14. Rekab K. A two-stage sequential allocation scheme for estimating the product of several means. Stochastic Analysis and Applications. 2000;18(2):289-298.

15. Rekab K, Li Y. Bayesian Estimation of the Product of Two Proportions. Stochastic Analysis and Applications. 1994;12(3):369-377.

16. Rekab K, Song X. Asymptotic Optimality of Three Stage Design for Estimating Product of Means with Applications in Reliability Estimation and Risk Assessment. Journal of Advances in Applied \& Computational Mathematics. 2016;3:8-19.

17. WoodroofeM.APORules areAsymptoticallynon-Deficient for Estimation with Squared Error Loss. Zeitschrift für Wahrscheinlichkeitstheorie und Verwandte Gebiete. 1981;58(3):331-341. 